\title{
LA VIOLENCIA COMO PROBLEMA DE SALUD PÚBLICA EN COLOMBIA: OTRO CAMPO PARA LA BIOÉTICA
}

\section{Favio Rivas M.}

Magíster en Medicina Tropical, Magíster en Epidemiología, Especialista en Bioética Actual representante del Comité Internacional de Rescate en Colombia

Correspondencia: Apartado Aereo 56053

Telefax (571) 2254994

E-mail: favrivas@hotmail.com

Bogotá - Colombia 


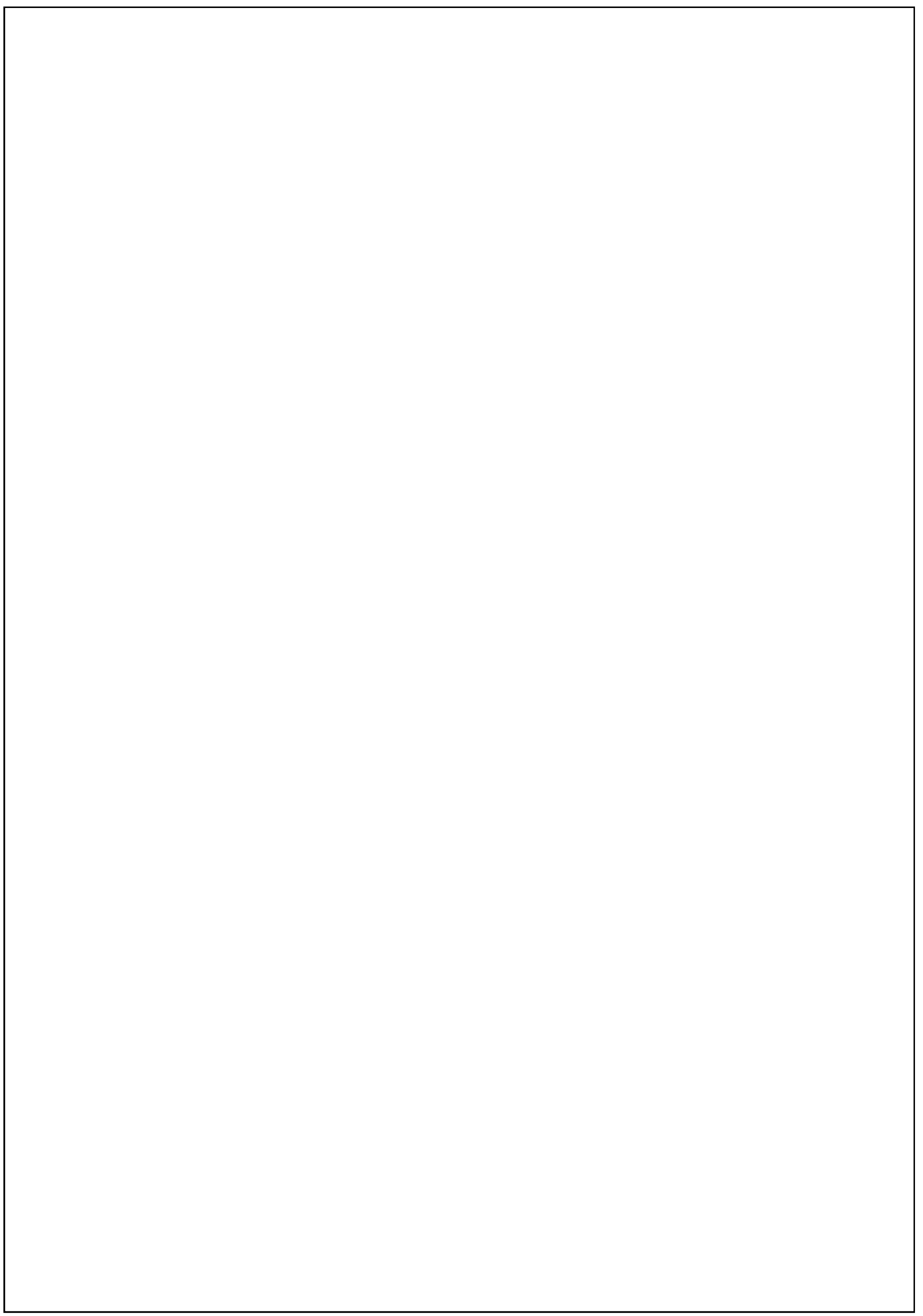




\section{LA VIOLENCIA COMO PROBLEMA DE SALUD PÚBLICA EN COLOMBIA: OTRO CAMPO PARA LA BIOÉTICA}

Favio Rivas M. ${ }^{(*)}$

\section{Resumen}

La violencia se viene incrementando a pesar de que el mundo ya no está polarizado. Con la caída del muro de Berlín muchos esperábamos el fin de las guerras, cosa que no ocurrió y, por el contrario, los conflictos internos en el tercer mundo son más y más frecuentes . Hoy, el homicidio en Colombia es la primera causa de muerte y son principalmente los hombres entre 15 y 44 años las principales víctimas. La violencia es hoy el mayor problema de salud pública para Colombia. Se trata de un asunto imposible de comprender desde un único punto de vista. Su abordaje, como la Bioética, debe ser interdisciplinario. Se trata de una circunstancia relacionada con la justicia distributiva, con la autonomía de los hombres y los pueblos, de un fenómeno donde prima la intención de hacer daño (maleficencia). Ahí hay un campo inmenso para la Bioética, sobretodo para quienes pensamos que es necesario sacarla del ambiente clínico a fin de aportar en la búsqueda de soluciones a problemas tan o más urgentes a este lado del mundo.

Palabras clave: Violencia, Salud Pública, Bioética
Resumo

A violência urbana cresce apesar de não mais haver polarização no mundo político. Com a queda do Muro de Berlim todos esperávamos o fim das guerras, coisa que não ocorreu, pelo contrário, os conflitos internos no terceiro mundo estão mais freqüentes. Atualmente, na Colômbia, o homicídio é a primeira causa de morte e faz dos homens entre 15 e 44 anos as principais vítimas. A violência constitui-se, hoje, como o maior problema de saúde pública da Colômbia. Trata-se de um assunto complexo que não se pode compreender sob um único ponto de vista. Sua abordagem, assim como a Bioética, deve ser interdisciplinar. Tratase de um fenômeno relacionado à justiça distributiva, com a autonomia dos homens e povos, onde prevalece a intenção de produzir dano( maleficência ). Existe, portanto, um imenso campo para a Bioética, sobre tudo para os que pensam ser necessário retirá-la do ambiente clínico e utilizá-la com instrumento de busca de soluções para os problemas mais urgentes neste lado do mundo.

Palavras chaves: Violência, Saúde Pública, Bioética.

(*) Presentación en el Curso Internacional de Bioética dentro del marco del VIII Congreso de la Asociación Latinoamericana de Medicina Social - Instituto de Ciencias Básicas y Preclínicas "Victoria de Girón”, 3 al 5 de julio del 2000. 


\begin{abstract}
Violence is still on the increase even though the world is no longer divided. With the fall of the Berlin wall most of us were expecting the end of wars, something that never happened. On the other hand, internal conflicts in the third world have become more and more frequent.

Today, homicide is the first cause of death in Colombia, being men aged from 15 to 44 the principal victims.

Violence is the major public health problem in Colombia nowadays. It is impossible to understand this issue from a unique point of view.

To address this problem, as it occurs with Bioethics, inter-disciplinary actions must be taken.

It is a phenomenon related to distributive justice and the autonomy of men and nation's, in a context where the intention to inflict harm (maleficence) prevails.

There exist, therefore, an enormous domain for bioethics, especially for those of us who deem necessary to remove it from its clinical atmosphere in order to collaborate in the searching for solutions to the urgent problems this side of the world is facing.
\end{abstract}

Key words: Violence, Public Health, Bioethics.

\section{Résumé}

La violence éprouve une croissance constante, bien que le monde ne soit plus polarisé. Après l'effondrement du mur de Berlin la plupart d'entre nous attendait la fin des guerres ce qui n'est jamais arrivé, au contraire, les conflits internes dans le tiers monde sont de plus en plus fréquents. Aujourd'hui, l'homicide en Colombie est la cause principale de décès et les victimes principales se sont essentiellement les hommes entre 15 et 44 ans. La violence est, de nos jours, le plus grand problème de santé publique pour la Colombie. Il s'agit d'une question impossible à comprendre d'un seul point de vue. L'approche de ce phénomène, de même que la Bioéthique, doit être interdisciplinaire. Il s'agit d'une circonstance liée à la justice distributive, à l'autonomie des hommes et des peuples, un phénomène où prévaut l'intention de faire du mal (malfaisance). Il y a ici un domaine très étendu pour la Bioéthique, surtout por ceux qui pensent qu'il est nécéssaire de la faire sortir de l'ambiance clinique afin de pouvoir faire des apports dans la recherche de solutions à des problèmes qui sont encore plus urgents, de ce côté du monde.

Mots clés: Violence, Santé Publique, Bioéthique. 
La violencia se ha tornado hoy en un problema de salud pública para el mundo entero. Se estima que, al paso que llevamos, hacia el año 2020, los homicidios ocuparán el décimo lugar entre las causas de muerte (1). En Colombia, sin embargo, desde 1990 y quizá un poco antes, es la primera (2). Es sabido que entre los 15 y los 44 años de edad el riesgo de morir es menor que en otras etapas de la vida y son fundamentalmente los accidentes o el SIDA los principales factores asociados, siendo los homicidios responsables de menos del $10 \%$ de esas muertes (1). En Colombia, por el contrario, alrededor del $80 \%$ de los muertos en ese grupo de edad, en el sexo masculino, fue asesinado (3). Las tablas anexas muestran las tasas de homicidio de diferentes países desarrollados y no. En ellas puede observarse como la de Colombia superó, de lejos, a cualquier otro país: 66.5 por $100 \mathrm{mil}$ habitantes en 1999 (4). Debe tenerse en cuenta que no se tiene estimativos acerca de la magnitud del subregistro.

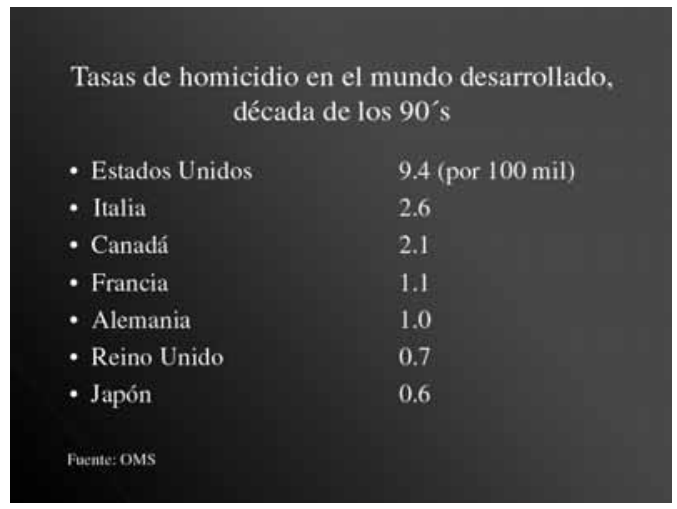

Hablando en números absolutos, en Colombia se mata anualmente una cifra similar a aquella de los Estados Unidos de Norteamérica (alrededor de 25.000 homicidios al año), pero ese país tiene una población siete veces mayor. Igualmente, el número de homicidios que anualmente ocurren en mi país es tres veces superior al de los ocurridos en la China, sólo que nuestra población es 30 veces menor.

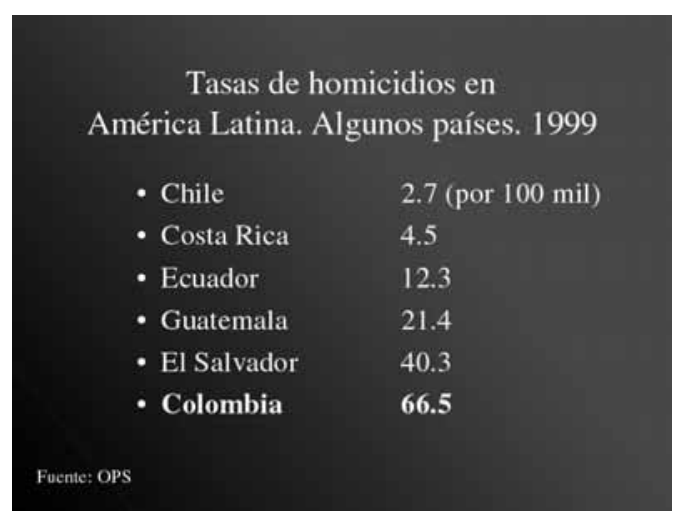

Las siguientes gráficas hacen referencia a dos de los actos más bárbaros en el contexto del conflicto colombiano: las masacres de personas en completo estado de indefensión por parte de cualquiera de los actores armados en conflicto, quienes en las noches o en las madrugadas, lista en mano, sacan de sus viviendas a personas que luego son asesinadas en presencia de sus familias, sin importar que haya niños, mujeres o ancianos y el desplazamiento forzado. Los datos son de la Defensoría del Pueblo, organismo de reciente creación y que vela por los derechos de los ciudadanos en Colombia (5). Las curvas van en ascenso.

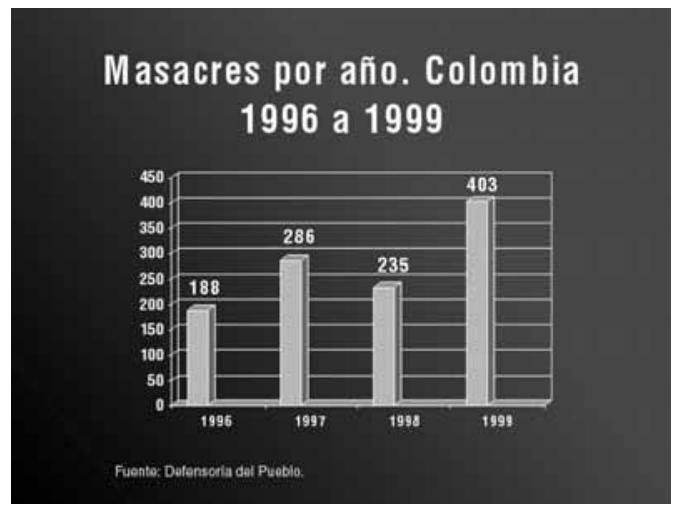

Uno de los indicadores utilizados para medir la carga de la enfermedad en los países son los Años de Vida Saludable (AVISA) perdidos. Pues bien, los homicidios responden por el $1.3 \%$ de esa carga en el mundo entero y por el 3.3\% en Latinoamérica. En Colombia, según el estudio acerca de la Carga de la Enfermedad, 
adelantado por el Ministerio de Salud en 1994, se pierde el $25 \%$ de los AVISA por dicha causa(6). Valga decir que hoy en Colombia 1 de cada 4 muertos lo fue por asesinato.

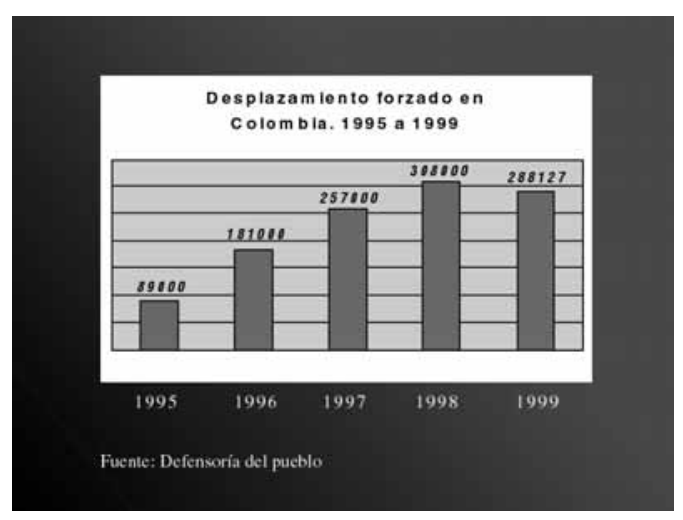

Esta información, cuyas fuentes aparecen al final de cada tabla o gráfica, es suficiente para entender que la violencia es el principal problema de salud pública hoy en Colombia. Como se dijo al comienzo, este problema no sólo es colombiano y es una tendencia general que ha suscitado interés $\mathrm{y}$, por tanto, se han dado distintas explicaciones a fin de lograr entender el fenómeno. Se ha dicho que en la base de todo conflicto social está la pobreza, la exclusión, la marginalidad, la intolerancia y las restricciones ciudadanas. Al respecto hay, desde posturas del Papa Juan Pablo II hasta "descubrimientos recientes" del Banco Mundial, que en uno de sus últimos informes refiere que la pobreza se perfila como el principal obstáculo para la salud pública en los países subdesarrollados (7).

También se han postulado, desde la academia, factores causales de tipo biológico como, por ejemplo, el papel de las hormonas masculinas, o se han hecho acercamientos desde lo psicológico (factores del comportamiento, de la socialización, cognitivos, etc.). Otros hablan de interacciones, como el uso de alcohol o estupefacientes por parte de sujetos con personalidades susceptibles de actos violentos. Hay también acercamientos desde las comunicaciones: el papel incitador y apologista de la violencia por parte de los medios (por algunos llamado efecto $\mathrm{CNN}$ ), en fin...hay abordajes desde diferentes ópticas y todos tienen alguna razón al igual que son válidos muchos de los intentos por lograr algún control del fenómeno, realizados desde esas miradas.

Kant decía que:

las sociedades no son nunca puro acuerdo ni pura discordia. Son siempre una ecuación entre ambas tensiones; una fuerza que tiende a la cohesión, que genera cooperación desinteresada, y otra que tiende a la disgregación. Así en el encuentro está el ideal normativo supremo de una comunidad de espíritus y en el otro la guerra de todos contra todos (8).

Es decir, a partir de este acercamiento desde la filosofía podemos ver que los conflictos sociales están siempre presentes y que no podemos pretender sociedades libres de ellos. En esa balanza, hoy es la guerra de todos contra todos la que parece estar viviéndose en Colombia y unos cuantos países más.

Para quienes llegamos a pensar alguna vez que la finalización de la llamada Guerra Fría traería como consecuencia la resolución de gran parte de los conflictos vividos a raíz de esa polarización del mundo entre los buenos del occidente y los malos del no-occidente, ha resultado al menos una sorpresa que se abriera esta "caja de Pandora" de los conflictos violentos al interior de muchos países. Esas fuerzas que tendían a la disgregación interna estaban "escondidas", subyacían silenciosas en medio de aguas aparentemente mansas y saltaron luego de derrumbarse el muro de Berlín. Hoy, los conflictos internacionales son la excepción y los que se dan dentro de las fronteras de país, son la regla.

La misma violencia que se observa en 
el resto del mundo, la de las calles de grandes ciudades, la dirigida contra la mujer o los niños, la asociada con el alcohol o las drogas, etc., se vive en Colombia, pero así mismo, a toda esa violencia se agrega aquí y en otros países de la región y del Tercer Mundo en general, la asociada a conflictos que siguen sin resolverse desde hace mucho tiempo.

William Ospina, en uno de los capítulos de un libro suyo editado en 1997 dice lo siguiente:

¿Cómo se sostiene una sociedad en la que todos saben que nada funciona? Desde los teléfonos que no sirven para hacer llamadas hasta los puentes que no sirven para ser usados y los funcionarios públicos que no sirven para atender a las personas y las fuerzas armadas que no sirven para defender la vida de los ciudadanos y los jueces que no sirven para juzgar y los gobiernos que no sirven para gobernar y las leyes que no sirven para ser obedecidas, el espectáculo que brindaría Colombia a un hipotético observador bienintencionado y sensato sería divertido si no fuera por el charco de sangre en que reposa (9).

Dentro de este panorama dónde nada funciona y quizá por ser este el panorama, es que también hay violencia. Este, a mi juicio, es un acercamiento al problema de violencia en Colombia, desde la literatura, como lo han hecho muchos excelentes escritores colombianos y el que los "científicos" no atendemos por ocuparnos de la exactitud o de la validez o de la representatividad.

Cuando nada sirve a los propósitos para los que fue concebido en la sociedad, se favorece la violencia. Que este sea un país de leyes nadie lo pone en duda, tenemos leyes para todo y, adicionalmente, nuestro Estado ha firmado infinidad de tratados y normas internacionales en materia de Derechos Humanos y otros asuntos, los cuales, simplemente no se tienen en cuenta. Por sólo poner un ejemplo, se escribió y así quedó aprobado en la Ley General de Seguridad Social en Salud, que el Estado colocaría un peso por cada peso que aportaran los contribuyentes al sistema, a fin de garantizar la atención de quienes no tienen la capacidad económica para costear sus gastos sanitarios (10). Pues bien, el Estado fue quién primero incumplió. ¿Dónde está su responsabilidad ética ante tamaño compromiso con los sectores más desprotegidos en materia de atención sanitaria? Aparte de pretender reemplazar con leyes y normas los vacíos éticos en nuestra sociedad, dichas leyes tampoco se cumplen, ni siquiera por quien las erigió como tales.

Desde la epidemiología, últimamente se han hecho muchos acercamientos al problema de violencia en Colombia. En 1997, en Indianápolis (EE.UU.), hubo un evento denominado Epidemiología Social de la Violencia. En él, el doctor Milton Terris hizo referencia a un estudio de casos y controles, adelantado en Medellín, bien diseñado y ejecutado por los investigadores en el cual "...al contrario de todas las expectativas: la prevalencia de desempleo era mayor en los controles que en los casos". Agrega el doctor Terris que:

El epidemiólogo que presentó el trabajo se apresuró a explicar este hallazgo peculiar: los asesinos tenían empleo, pues trabajaban para el cartel de la droga de Medellín (11).

Hoy en día el desempleo en Colombia supera el $20 \%$ y el "sector violencia" es ahora una alternativa de ocupación cuando alguien se queda cesante. Juan Manuel López Caballero en su libro La violencia de los 90's muestra una tabla, que a continuación se observa, la cual se modificó en su formato para esta presentación (12). 


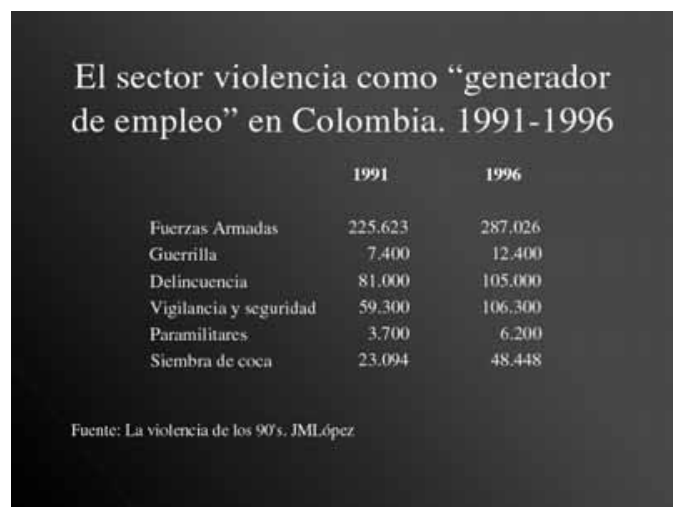

Si se compara los años 1991 a 1996, el sector violencia ha venido incrementando grandemente la cantidad de sus "empleados". Vaya sociedad!... las exigencias de "eficiencia" desde la óptica de la economía han dejado sin empleo a miles y miles de personas, muchas de quienes, al no encontrar nada mejor, se suman a las filas de los violentos, los que si tienen suficiente dinero para pagar buenos salarios. Estos "empleados" realizan su "trabajo" con sorprendente eficiencia, pues muy pocos son atrapados (¿será por ser crímenes perfectos?) y gran parte de los atrapados logra evadir la justicia.

Para seguir con abordajes desde la economía, el autor anteriormente citado refiere que:

En todos los países existen franjas de población pobre sin que ellas, ya sea como franjas o como personas, se caractericen por ser violentas o peligrosas (por el contrario, sorprende su tendencia a la resignación).

Hay, sin duda, países con tantos y aún mucho más pobres que Colombia, pero ninguno tan violento. Quizá tengan razón quienes argumentan que más que la pobreza es la desigualdad, es decir, de una lado la opulencia y del otro la miseria, con una distancia cada vez mayor entre los dos extremos.
La tabla que sigue, muestra la diferencia entre la proporción del Producto Interno Bruto (PIB) que recibe la quinta parte más rica de la población colombiana con respecto a la correspondiente para la quinta parte más pobre. Si bien son altos estos indicadores para el África Subsahariana y para Latinoamérica y el Caribe con respecto al resto del mundo, tal diferencia para Colombia es aún mayor (13).

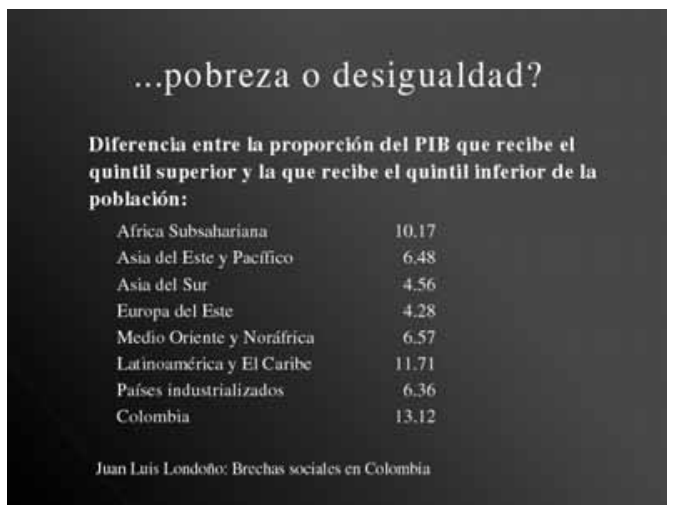

Dejando de lado la economía y las frías estadísticas -que tanto nos ayudan a quienes trabajamos en salud pública, pero que también han sido responsables, en parte, de la corta visión y de la crisis en que hemos "patinado" los salubristas en los últimos años- vale la pena entrar un poco en la experiencia de la modernidad. Al respecto, y muy brevemente, podríamos decir que hemos vivido (y seguimos viviéndola!) el proceso de modernización pues el Estado Colombiano es liberal en lo económico, pero la modernidad si que no se ha dado a la par con ese proceso; es conservador en lo político. Claro, esto no ha llevado a conflictos entre los dos actores quienes, al contrario, se articulan y se prestan mutuos servicios (14). Son los intereses de estos individuos y no los de la sociedad colombiana los que han primado históricamente. Nuestras democracias son, como decía Estanislao Zuleta (escritor, profesor universitario y psicoanalista ya fallecido), "barcos cuya tripulación guía a puertos seguros pero cuyos pasajeros sabemos, son puertos donde la tripulación y sus familias tienen negocios". 
La modernización ha sido un fin supremo de nuestro estado desde el momento mismo en que en Europa se estaba viviendo el proceso como un medio hacia mejores condiciones materiales de vida. Es decir, lo que para Europa era un camino hacia un mundo mejor y se caminaba con los propios pies, para nuestros países era una meta que no se sabía si iba a funcionar....y, efectivamente, en muchos no ha funcionado!

Hoy, lo moderno es la sociedad occidental sin pensar que hay sociedades occidentales al norte, al sur, al este y al oeste, con mucho o poco parecido unas y otras y con diferencias en la experiencia de la modernidad. Es decir que ni lo moderno ni lo occidental son hoy universales. En este occidente de colores, a unas sociedades les fue impuesta la modernidad desde arriba, se hizo por la fuerza y no fue un proceso que surgiera del entendimiento de sus necesidades y sus intereses como pueblos. Se trataba de llegar a una meta sin recorrer un camino, al menos uno caminado con los propios pies. Así resultaron unas instituciones y un discurso político formalmente modernos, basados en la igualdad, la libertad y la fraternidad, sobre unos cimientos totalmente envejecidos. La libre competencia del mercado no se ha acompañado de la libre competencia de las ideas ni de la apertura de oportunidades para que ella se de. Lo moderno, visto como medio, habría de ser caminado por las sociedades y los pueblos a su manera. América Latina entró a la modernidad sin haberse aventurado a recorrerla, hizo una copia en el papel de aquello que vivieron (y padecieron!) poco a poco otros países. Se transformaron las cosas y se privilegiaron sobre los hombres. Este privilegiar de las cosas avanza a pasos agigantados sin que los seres humanos nos transformemos. Como en el mito de Sísifo, quien fue condenado a subir una piedra hasta la cima de una loma y esta rodaba para que Sísifo la volviera a subir y volviera a rodar, estamos siendo condenados a trabajar para poder consumir y desechar y volver a trabajar para seguir consumiendo y desechando...

Quizá, cuando comenzaba la modernización en nuestro territorio, los ricos, armados y poderosos eran los mismos y había que matar a quienes nada de eso eran o tenían; luego fueron apareciendo otros ricos, aunque sin armas y sin poder, a buscarlo; no los dejaron y por tanto era "necesario" matar; más tarde unos no ricos pero armados y sin poder, vinieron a buscarlo y, como siempre también había que matar. En Colombia, parodiando a Facundo Cabral (cantautor argentino), los verdes pelean contra los amarillos para defender la tierra que los naranja arrebataron a los grises. Aquí se mata por la justicia, por la libertad, por el orden, por las buenas costumbres, por unos zapatos Adidas, por una billetera, por un pan, por ganar, por perder, por empatar...Aquí se te mata por hacer uso público de la razón cuando la tienes pero "atenta" con la visión de alguno o algunos que dicen también tenerla. Los oponentes ahora son enemigos, tu vida depende de lo que pienses o de lo que opines, de lo que tengas o te falte. Eres un medio y los fines no te contemplan.

Se habla de que vivimos una cultura de la violencia, pero eso no puede ser cultura. Más bien se trata de una cultura que la incita y la estimula, una cultura sustentada en la lucha de todos contra todos, donde sobreviven lo más aptos, es decir, los más poderosos por su riqueza o por sus armas, o por las dos al tiempo, o los más avivados, o los más rápidos o los más "modernos". ¿No es esta una cultura del crimen? Hoy no hace falta el cerebro si tu computador dispone de un disco duro de gran capacidad. Hoy no hace falta el corazón, late muy lento, es necesario el más veloz procesador. Hoy el sentimiento y la razón están sobrando.

Hace unos cuantos años leí Memoria del Fuego, de Eduardo Galeano, donde él cuenta de un misionero español que trataba de persuadir de las bondades del Dios de los 
católicos a un Jefe Indio. Luego que el misionero habló, el indio le dijo algo como: "eso rasca, rasca mucho, rasca muy bien...pero rasca donde no pica" (15). La bioética ha permitido un reencuentro entre los valores humanísticos y científicos, pero las prioridades en materia de conflictos de los que debería ocuparse no son las mismas para todas las sociedades y las culturas. Lo que es un conflicto frecuente al occidente del norte, no lo es al occidente del sur o al del este o al del oeste. En estos otros occidentes estamos en el deber de no seguir copiando. Tenemos problemas de salud y de salud pública que pueden parecerse pero al menos su frecuencia es diferente. Tenemos problemas de justicia distributiva y sanitaria que no son los mismos.

La salud, vista como la "magnitud en que un individuo o grupo pueden, por una parte, realizar sus aspiraciones y satisfacer sus necesidades, y por otra, cambiar su entorno o afrontarlo", como aparece en un texto de OPS de 1996, va más allá de la recuperación del herido o el enfermo, va más allá del acceso a los servicios y no separa lo físico de lo mental o lo social (16). La bioética, aunque haya nacido $y$ prácticamente se haya criado en un medio hospitalario y científico, debe, además de estar ahí, salir de los hospitales y de los bancos de tejidos u órganos, de los comités de ética hospitalarios y de ética de la investigación, de los centros de genética,...para que al menos rasque más donde más está picando.

Si la violencia, como problema de salud pública, requiere para su abordaje en busca de respuesta del encuentro de múltiples disciplinas, entonces hay espacio y tiempo aún para la bioética. Si la violencia es una forma de relación entre los humanos con intención de hacer daño (maleficencia), sin tener en cuenta la autonomía del otro, entonces es una campo donde cabe la bioética. Si la violencia tiene que ver con la inequidad (falta de justicia), tiene que ver con la bioética. Si la violencia atenta contra el valor supremo del hombre como fin y no como simple medio hacia fines considerados de mayor importancia, debe ser abordada por quienes nos interesamos por la bioética.

En política, decía Albert Camus, los fines no justifican los medios y, al contrario, los medios deberían justificar esos fines. Desde ese punto de vista, resulta difícil persuadir a las personas de la justeza de unos fines cuando los medios utilizados son bárbaros y brutales. Si la guerra, al decir de muchos, es una extensión de la política, la justeza de los medios resulta una fortaleza en lo ético y político. Lo que está detrás de esa cantidad de normas del Derecho Internacional Humanitario (DIH) es esa fuerza ética y política que parecen no ver los actores armados en el conflicto colombiano. Respetar el DIH sería uno de esos mínimos éticos (que por serlo se tornan políticos) necesarios y urgentes en el marco del conflicto colombiano a fin de encontrar salidas a la barbarie.

Desde luego que se deben resolver los conflictos cotidianos, las querellas callejeras, las disputas familiares, etc., por vías no violentas, mediante el diálogo, mediante acuerdos. Desde luego que es necesario ir al rescate de valores perdidos por dedicarnos a vivir para el consumo y la competencia egoísta. Pero además, particularmente en Colombia, se deben atender los conflictos que atañen al Estado, como el de la tenencia de la tierra, el de un empleo digno, el de un salario que al menos cubra el costo de una canasta familiar, los relacionados con esas minorías indígenas y su visión del progreso, del ambiente, de la vida, en fin, conflictos que secularmente se dejaron a merced del tiempo y no de los hombres que pudieron y tuvieron en sus manos la respuesta. Los privilegiados de siempre deberán ceder un poco de esos privilegios $(17,18)$.

Por no tener respuestas debo hacer, como hizo Saúl Franco al final de su libro $E l$ quinto: No matar, unas "inconclusiones". 
Empiezo diciendo que las utopías eran y siguen siendo, medios. Las utopías aún permanecen intactas como caminos que deberemos recorrer a fin de no perder el rumbo, sean utopías en lo individual o en lo colectivo. Como medios o caminos, no podemos delegar en otros pies el acto de recorrerlos, deben ser justos, deben ser cuidados por nosotros.

Esta sociedad moderna es la del presente, un presente tan largo y tan urgente que nos arrebató la capacidad para reflexionar en el futuro y ni siquiera del pasado obtuvimos experiencias. ¿Será, como escribe William Ospina, que ya es tarde para el hombre? (19).

¿Quedarán hombres justos?; si quedan ¿les dejarán opinar?; si les dejan, ¿les permitirán gobernar?; si les permiten, ¿los apoyaremos?, ¿estaremos dispuestos a aprender de ellos?

Es tal la velocidad y tanta la cantidad de información y de conocimiento científico y técnico, que no tenemos tiempo ni espacio para la sabiduría, para la virtud. No planteo que se requiera de un sabio, hoy, más que nunca antes, se requieren millones de ellos, pero ¿Cómo hacer hombres justos y virtuosos cuando hay tantos hambrientos, cuando hay tantos analfabetos, cuando mueren tantos niños y matan tantos jóvenes en este occidente del sur que, como el del norte, sólo se ocupa de los índices económicos y de favorecer a los siempre privilegiados?

En Colombia, dice William Ospina, "los pobres no pueden comer, la clase media no puede comprar y los ricos no pueden dormir" (20). Históricamente los pobres no han podido comer y esto muy poco ha cambiado porque muy poco ha importado a los privilegiados de todas las épocas. Pero que en la sociedad de hoy, la clase media, empujada al consumo por todos los medios, no pueda comprar y que los que siempre han podido comprar, vender y dormir, ya no puedan hacerlo tranquilos, parece ser el caos total. Al menos algo anda mal y algo habrá que hacer porque, como está la sociedad, no habrá futuro para nadie:

Llevamos siglos desobedeciendo códigos perfectos, transgrediendo normatividades admirables y vanagloriándonos de la perfección de unas instituciones a las que nadie respeta, plantea Ospina (20).

La modernidad no ha llegado a pesar de esa perfección de los códigos, las normatividades y las instituciones. Deberemos caminarla con lo que somos, con lo que tenemos y nos falta.

\section{Referencias}

1. Rosenberg ML, Mercy JA, Annest JL. The problem of violence in the United States and globally. In: Public Health and Preventive Medicine. $14^{\text {th }}$ Ed. London: Appleton \& Lance; 1998. p.1223-6.

2. Instituto Nacional de Salud, Ministerio de Salud de Colombia. La violencia en Colombia: un problema que el sector salud debe reconocer y afrontar. Informe Quincenal Epidemiológico Nacional 1998;2(3): 2-3.

3. Franco S. El Quinto: No matar. Bogotá: Tercer Mundo Editores; 1999.

4. Organización Panamericana de la Salud / Organización Mundial de la Salud. Indicadores Básicos de Salud; Washington D.C.: OPS/OMS;1999.

5. Defensoría del Pueblo. Masacres en Colombia 1990 - 1999. Bogotá: Carrera Séptima Ltda.;1999.

6. Ministerio de Salud. La Carga de la Enfermedad en Colombia. Bogotá: Carrera Séptima Ltda.;1994.

7. Banco Mundial. Informe sobre el Desarrollo Mundial. Ginebra: Banco Mundial; 1999.

8. Kant M. Fundamentación de la metafisica de las costumbres: crítica de la razón práctica. $9^{a}$ ed. México: Porrúa, S.A.; 1996.

9. Ospina W. ¿Dónde está la franja amarilla?. Santafé de Bogotá: Norma; 1997. 
La violencia como problema de salud pública en Colombia: otro campo para la Bioética - F. Rivas

10. Ley $N^{o} 100$. Ley General de Seguridad Social en Salud. Santafé de Bogotá: Ministerio de Salud;1993.

11. Terris M. Violencia en una sociedad violenta. Traducción libre de Helena Restrepo. Revista Facultad Nacional de Salud Pública 1999; 17(1): 11-15.

12. López JM. La violencia de los 90's. Santafé de Bogotá: Gráficas Ducal Ltda; 1998.

13. Londoño JL. Brechas Sociales en Colombia. Bogotá: Tercer Mundo Editores; 1997.

14. Corredor C. Los límites de la modernización. $2^{a}$ ed. Bogotá: Universidad Nacional de Colombia; 1997.

15. Galeano E. Memoria del Fuego: I. Los nacimientos. La Habana: Casa de las Américas; 1988.

16. Organización Panamericana de la Salud.
Promoción de la Salud: una antología. Washington, D.C.: OPS; 1996. ( Publicación científica 557).

17. Rivas F. La salud mental en el contexto de violencia y desplazamiento forzoso en Colombia: un desafío a la salud pública hoy y mañana. En: Bello M, Martín E, Arias F, (eds). Efectos psicosociales y culturales del desplazamiento. Bogotá: UNIBIBLOS; 2000 p. 78-82.

18. Rivas F. Vida y muerte violentas: el desafío más grande para la bioética en Colombia. Revista Médica de Risaralda 2000; 6(1):3045.

19. Ospina W. ¿Es tarde para el hombre? $3^{\mathrm{a}} \mathrm{ed}$. Santafé de Bogotá: Norma; 1999.

20. Ospina W. Lo que le falta a Colombia. Santafé de Bogotá: Norma; 1999. 